\title{
Near-Quantitative Triplet State Population via Ultrafast Intersystem Crossing in Perbromoperylendiimide
}

Amalu Mohan ${ }^{\ddagger}$, Ebin Sebastian ${ }^{\ddagger}$, Mahesh Gudem and Mahesh Hariharan*

₹ Th ese authors contributed equally to this work

School of Chemistry, Indian Institute of Science Education and Research Thiruvananthapuram (IISER-TVM), Maruthamala P.O., Vithura, Thiruvananthapuram 695551, Kerala, India. E-mail: mahesh@iisertvm.ac.in

\section{Supporting Information}

\section{Contents}

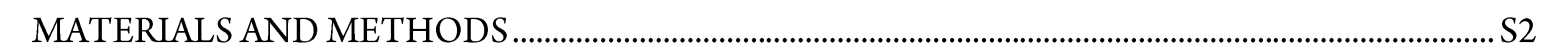

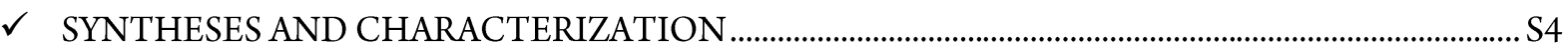

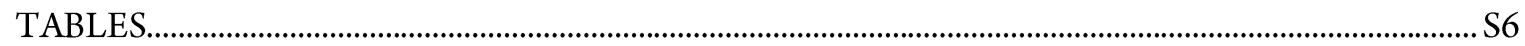

- Table S1: Crystal data and structure refinement parameters of OBPDI crystal................................... S6

- Table S2: Electrochemical studies of derivatives of OBPDI and PDI ...................................................... S6

- Table S3: Vertical excitation energies and oscillator strengths of OBPDI geometry for ten lowest singlet and triplet excited states at TDDFT/B3LYP/6-31G(d,p) level of theory.

- Table S4: Vertical excitation energies and oscillator strengths of PDI and PDI-Twisted geometries for four lowest singlet excited states at TDDFT/B3LYP/6-31G(d,p) level of theory.

- Table S5. Spin-orbit coupling values between $S_{1}$ and low-lying triplet states at PDI minimum and PDI-Twisted geometries evaluated at TDDFT/B3LYP/6-31G(d,p) level of theory.

- Table S6: A comparative account of populating the triplet excited state in PDIs by covalent methods.

- Figure S1: ORTEP image OBPDI crystallized from benzene........................................................................ S9

- Figure S2: Cyclic Voltammogram of OBPDI and PDI.......................................................................... S9

- Figure S3: Fluorescence emission spectra of OBPDI..............................................................................

- Figure S4: Fluorescence decay profiles of OBPDI and PDI..................................................................S10

- Figure S5: The decay traces at the triplet maxima under $\mathrm{N}_{2}$ and $\mathrm{O}_{2}$ purged conditions in OBPDI.S10

- Figure S6: Graphical representation of trend in the fluorescence $\left(\Phi_{1}\right)$ and triplet $\left(\Phi_{1}\right)$ quantum yields with increase in the number of bromine atoms (from $\mathrm{Br}_{0}-\mathrm{PDI}\left(0 ; \mathrm{x}\right.$-axis), $\mathrm{Br}_{1}$-PDI ( 1 ; $\mathrm{x}$-axis), $\mathrm{Br}_{2}$ PDI (2; x-axis), $\mathrm{Br}_{3}$-PDI (3; x-axis), $\mathrm{Br}_{4}$-PDI (4; $\mathrm{x}$-axis) and OBPDI/Br 8 -PDI (8; $\mathrm{x}$-axis)

- Figure S7: Graphical representation for vertical energies of lowest singlet and triplet excited states of OBPDI (computed at TDDFT/B3LYP/6-31G (d,p) level of theory). 
- $\mathrm{S}_{0}-\min$ for OBPDI at TDDFT/B3LYP/6-31G(d,p)

- $\quad \mathrm{S}_{0}$-min for PDI at TDDFT/B3LYP/6-31G(d,p)

\section{MATERIALS AND METHODS}

All chemicals were purchased from commercial suppliers and used as received without further purification. All the reactions were carried out in glassware dried in oven before using and wherever necessary, were performed under dry nitrogen in dried, anhydrous solvents using standard gastight syringes, cannulae, and septa. Solvents were dried and distilled by following the standard procedures. TLC analysis were executed on precoated aluminum plates of silica gel $60 \mathrm{~F} 254$ plates $(0.25 \mathrm{~mm}$, Merck) and developed TLC plates were viewed under short and long wavelength UV lamps. Flash column chromatography was performed using silica gel of 200-400 mesh employing a solvent polarity correlated with the TLC mobility observed for the molecule of interest. Yields refer to chromatographically and spectroscopically homogenous substances. To get a rough estimate, the melting points (MP) were initially determined using a capillary melting point apparatus. ${ }^{1} \mathrm{H}$ and ${ }^{13} \mathrm{C}$ NMR spectra were measured on a $500 \mathrm{MHz}$ Bruker advanced DPX spectrometer. Internal standard used for ${ }^{1} \mathrm{H}$ and ${ }^{13} \mathrm{C}$ NMR is 1,1,1,1-tetramethyl silane (TMS). High resolution mass spectra (HRMS) were recorded on Thermo Scientific Q Exactive mass spectrometer using electrospray ionization (ESI, positive mode) technique.

\section{Calculating Radiative $\left(\boldsymbol{k}_{r}\right)$ and Non-Radiative $\left(\boldsymbol{k}_{\mathrm{nr}}\right)$ Rate Constants}

The radiative $\left(k_{\mathrm{r}}\right)$ and non-radiative $\left(k_{\mathrm{nr}}\right)$ rate constants for OBPDI and PDI were calculated using the equations 3 and $4 .{ }^{1,2}$

$$
\begin{gathered}
\phi_{\mathrm{f}}=\frac{k_{\mathrm{r}}}{k_{\mathrm{r}}+k_{\mathrm{nr}}} \\
\tau_{\mathrm{f}}=\frac{1}{k_{\mathrm{r}}+k_{\mathrm{nr}}} \\
k_{r}=\frac{\phi_{\mathrm{f}}}{\tau_{\mathrm{f}}} \\
k_{\mathrm{nr}}=\frac{1}{\tau_{\mathrm{f}}}-k_{\mathrm{r}}
\end{gathered}
$$

Where $\phi_{f}$ the fluorescence quantum is yield and $\tau_{\mathrm{f}}$ is the fluorescence lifetime. In case of multi-exponential decay, the weighted average of the fluorescence lifetime values was used for estimation of rates of radiative and non-radiative processes using equation 5,

$$
\tau_{\mathrm{f}}=\frac{\alpha_{1} \tau_{1}^{2}+\alpha_{2} \tau_{2}^{2}+\alpha_{3} \tau_{3}^{2}+\ldots}{\alpha_{1} \tau_{1}+\alpha_{2} \tau_{2}+\alpha_{3} \tau_{3}+\ldots}
$$

Where $\alpha=$ amplitude corresponding to the fluorescence lifetime $\left(\tau_{\mathrm{f}}\right)$ decay. 


\section{Calculating the Rate of Intersystem Crossing $\left(\boldsymbol{k}_{\text {ISC }}\right)$}

The rate of intersystem crossing $\left(k_{\mathrm{ISC}}\right)$ was estimated from the efficiency of intersystem crossing $\left(\phi_{\mathrm{ISC}}\right)$ and fluorescence lifetime $\left(\tau_{\mathrm{f}}\right)$ as follows, ${ }^{3}$

$$
\begin{gathered}
\phi_{\mathrm{ISC}}=\frac{k_{\mathrm{ISC}}}{k_{\mathrm{r}}+k_{\mathrm{nr}}} \\
k_{\mathrm{total}}=k_{\mathrm{r}}+k_{\mathrm{nr}}=1 / \tau_{\mathrm{f}} \\
\phi_{\mathrm{ISC}}=k_{\mathrm{ISC}} \tau_{\mathrm{f}} \\
k_{\mathrm{ISC}}=\frac{\phi_{\mathrm{ISC}}}{\tau_{\mathrm{f}}}
\end{gathered}
$$

Where $\tau_{\mathrm{f}}$ is the fluorescence lifetime and $\phi_{\mathrm{ISC}}$ is the efficiency of intersystem crossing.

\section{X-ray crystallography}

High-quality specimens having appropriate dimensions of OBPDI were selected for the X-ray diffraction experiments. Crystallographic data collected are presented in the supplementary information. Single crystals were mounted using oil (Infineum V8512) on a glass fibre. All measurements were made on a CCD area detector with graphite monochromated $\mathrm{Mo}_{a}$ radiation. The data was collected using Bruker APEXII detector and processed using APEX2 from Bruker. Structure was solved by direct methods and expanded using Fourier techniques. The non-hydrogen atoms were refined anisotropically. Hydrogen atoms were included in idealized positions, but not refined. Their positions were constrained relative to their parent atom using the appropriate HFIX command in SHELXL-97. 


\section{SYNTHESES AND CHARACTERIZATION}
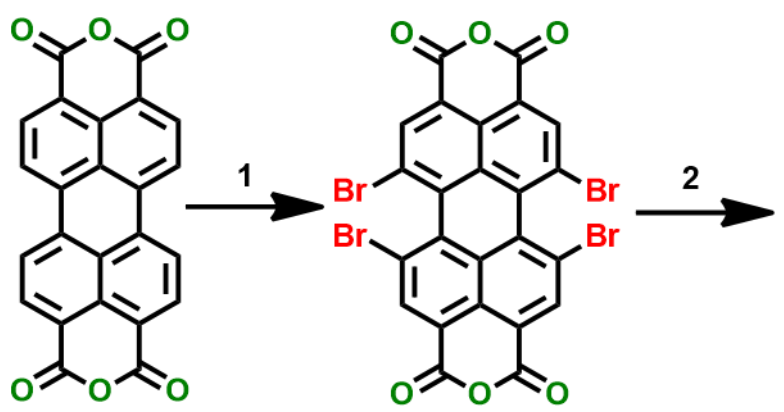<smiles>O=c1oc(=O)c2c(=O)oc(=O)c3c(Br)c(Br)c4c(Br)c(Br)c5c(Br)c(Br)c1c1c(Br)c(Br)c2c3c4c51</smiles>
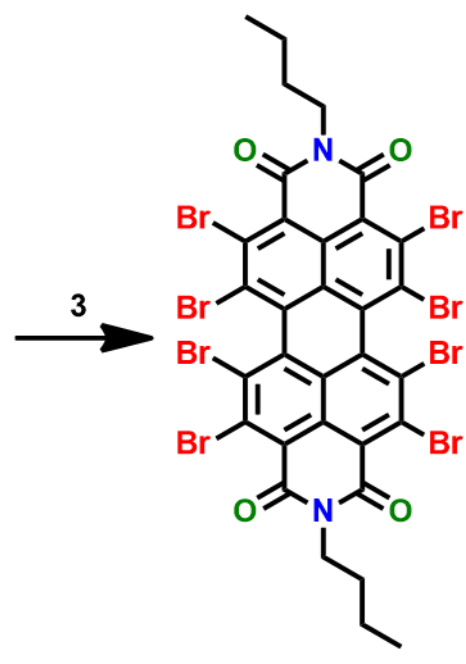

$1 \mathrm{Br}_{2}$, Con. $\mathrm{H}_{2} \mathrm{SO}_{4}, \mathrm{I}_{2}, 110^{\circ} \mathrm{C}$

$2 \mathrm{DBH}, \mathrm{Con} . \mathrm{H}_{2} \mathrm{SO}_{4} 110^{\circ} \mathrm{C}$

3 N-Butylamine, $\mathrm{DCM} \mathrm{PBr}_{3}$

Scheme S1. Shows the synthesis of OBPDI. The syntheses scheme was taken from the reported procedure. ${ }^{4}$

\section{Synthesis of 1,6,7,12-tetrabromoperylene-3,4,9,10-tetracarboxylic dianhydride (PTCDA-Br 4 )}

In a $250 \mathrm{ml}$ round-bottom flask, $2.5 \mathrm{~g}$ of perylene-3,4,9,10-tetracarboxylic dianhydride (PTCDA) was stirred with $100 \mathrm{ml}$ of concentrated sulphuric acid for 5 hours. Catalytic amount of elemental iodine was added and the reaction mixture was heated to $110^{\circ} \mathrm{C} .4 .4$ equivalence of elemental bromine was added dropwise into the

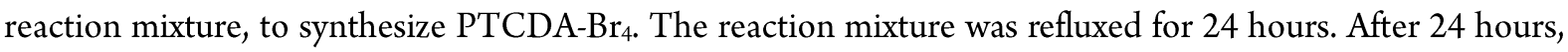
the product was precipitated by pouring the reaction mixture into crushed ice. The resultant precipitate was filtered through a Buchner funnel, washed with water and methanol. The solid was dried in hot air oven to yield PTCDA-Br $r_{4}$ as red solid. Owing to the insolubility of the compound, it was taken for the next step of synthesis without any characterization.

\section{Synthesis of 1,2,5,6,7,8,1 1,12-octabromoperylene-3,4,9,10-tetracarboxylic dianhydride (OBPDA)}

$1.5 \mathrm{~g}$ of PTCDA-Br$r_{4}$ was weighed into a $250 \mathrm{ml}$ round-bottom flask. $50 \mathrm{ml}$ of Conc. $\mathrm{H}_{2} \mathrm{SO}_{4}$ was added and stirred for 15 minutes at RT. The temperature was then raised to $110^{\circ} \mathrm{C}, 1.5 \mathrm{~g}(5 \mathrm{mmol})$ of DBH (1, 3-dimethyl-5, 5-dibromohydantoin) was added in portions over a period of 30 minutes. The reaction mixture was refluxed for 24 hours. After 24 hours, the product was precipitated by pouring the reaction mixture into crushed ice. The resultant precipitate was filtered through a Buchner funnel, washed with water and methanol. The solid was dried in hot air oven to yield OBPDA as red solid. Yield $=70 \%$. Melting Point $>300^{\circ} \mathrm{C}$.

${ }^{13} \mathbf{C}$ NMR (125 MHz, $\left.\mathrm{CDCl}_{3}, \mathrm{TMS}\right): 156.55,135.06,133.04,131.31,128.27,127.88,120.22$

HRMS (ESI): $\mathrm{m} / \mathrm{z}$ calculated for $\mathrm{C}_{24} \mathrm{Br}_{8} \mathrm{O}_{6}[\mathrm{M}+\mathrm{Na}]^{+}: 1046.4852$, found 1046.4858 


\section{Synthesis of 1, 2, 5, 6, 7, 8, 11, 12-octabromo perylene-3, 4, 9, 10-tetracarboxylic diimide (OBPDI)}

OBPDA ( $250 \mathrm{mg}$ ) was taken in a $100 \mathrm{ml}$ round-bottom flask, $30 \mathrm{ml}$ of DCM was added. It was stirred for 10 minutes at RT. N-Butylamine ( $2.5 \mathrm{eq}$ ) was added dropwise, refluxed for 2 hours. After 2 hours $\mathrm{PBr}_{3}$ (1eq) was added and further refluxed for $1 \mathrm{~h}$. The reaction was cooled to room temperature and $100 \mathrm{ml}$ of water was added. Aqueous layer was separated using DCM. The organic layer was collected and solvent evaporated under reduced pressure. The crude mixture was purified by column chromatography with DCM: hexane (1:1) Yield $=70 \%$. Melting point $>300^{\circ} \mathrm{C}$.

${ }^{1} \mathrm{H}$ NMR $(500 \mathrm{MHz}, \mathrm{CDCl} 3, \mathrm{ppm}): \delta=4.27-4.21(\mathrm{~m}, 2 \mathrm{H}), 4.17-4.12(\mathrm{~m}, 2 \mathrm{H}), 1.72-1.66(\mathrm{~m}, 4 \mathrm{H}), 1.44-1.39$ $(\mathrm{m}, 4 \mathrm{H}), 0.94(\mathrm{t}, \mathrm{J}=7.5,6 \mathrm{H})$.

${ }^{13}$ C NMR: (125 MHz, CDCl3, TMS): $\delta=160.10,133.42,132.78,132.12,127.60,125.83,121.72,42.29$, $31.06,20.64,13.56$.

HRMS (ESI): $\mathrm{m} / \mathrm{z}$ calculated for $\mathrm{C}_{32} \mathrm{H}_{18} \mathrm{Br}_{8} \mathrm{~N}_{2} \mathrm{O}_{4}[\mathrm{M}+\mathrm{Na}]^{+}: 1156.7283$ found: 1156.7397 . 


\section{TABLES}

Table S1: Crystal data and structure refinement parameters of OBPDI crystal.

\begin{tabular}{|c|c|}
\hline Unit cell parameters & \\
\hline Empirical formula & $\begin{array}{c}\mathrm{C}_{32} \mathrm{H}_{18} \mathrm{Br}_{8} \mathrm{~N}_{2} \mathrm{O}_{4}, \\
2\left(\mathrm{C}_{6} \mathrm{H}_{6}\right)\end{array}$ \\
\hline Formula weight & 1289.98 \\
\hline Colour, shape & Red, needle \\
\hline Crystal system & Monoclinic \\
\hline Space group & $\mathrm{C} 2 / \mathrm{c}, 4$ \\
\hline $\mathrm{A}(\AA)$ & $21.043(4)$ \\
\hline $\mathrm{B}(\AA)$ & $7.3051(16)$ \\
\hline $\bar{C}(\AA)$ & $28.007(6)$ \\
\hline$\alpha, \operatorname{deg}$ & 90 \\
\hline$\beta, \operatorname{deg}$ & $105.827(8)$ \\
\hline$\lambda, \operatorname{deg}$ & 90 \\
\hline Volume $\left(\AA^{3}\right)$ & 4142.06 \\
\hline Temperature (K) & 296 \\
\hline Calculated density $\left(\mathrm{mg} / \mathrm{m}^{3}\right)$ & 2.069 \\
\hline Reflections collected & 16791 \\
\hline Unique reflections & 4052 \\
\hline Number of parameters & 251 \\
\hline $\mathrm{R}_{1}, \mathrm{wr}_{2}(\mathrm{i}>2 \sigma(\mathrm{i}))$ & $0.0533,0.1501$ \\
\hline $\mathrm{R} 1, \mathrm{wr}_{2}\left(\mathrm{f}^{2}\right)$ (all data) & $0.0872,0.1733$ \\
\hline $\mathrm{R}(\mathrm{f} \%)$ & 5.33 \\
\hline Goodness of fit on $\mathrm{f}^{2}$ & 1.004 \\
\hline CCDC number & 1913335 \\
\hline
\end{tabular}

Table S2: Electrochemical studies of derivatives of OBPDI and PDI

\begin{tabular}{|c|l|c|}
\hline Derivative & $\mathrm{E}_{\text {red1 }}(\mathrm{V})$ & $\mathrm{E}_{\text {red2 }}(\mathrm{V})$ \\
\hline OBPDI & -0.170 & -0.341 \\
\hline PDI & -0.547 & -0.728 \\
\hline
\end{tabular}


Table S3: Vertical excitation energies and oscillator strengths of OBPDI geometry for ten lowest singlet and triplet excited states at TDDFT/B3LYP/6-31G(d,p) level of theory.

\begin{tabular}{|c|c|c|c|c|}
\hline \multicolumn{4}{|c|}{ States } & \multirow{2}{*}{$\begin{array}{l}\text { Oscillator } \\
\text { Strength } \\
\text { (f) }\end{array}$} \\
\hline Singlet & $\begin{array}{c}\text { Energy } \\
(\mathrm{eV})\end{array}$ & Triplet & $\begin{array}{c}\text { Energy } \\
(\mathrm{eV})\end{array}$ & \\
\hline$S_{1}$ & 2.264 & $\mathrm{~T}_{1}$ & 1.347 & 0.3269 \\
\hline $\mathrm{S}_{2}$ & 2.355 & $\mathrm{~T}_{2}$ & 2.126 & 0.0113 \\
\hline $\mathrm{S}_{3}$ & 2.421 & $\mathrm{~T}_{3}$ & 2.192 & 0.0006 \\
\hline $\mathrm{S}_{4}$ & 2.635 & $\mathrm{~T}_{4}$ & 2.252 & 0.1414 \\
\hline $\mathrm{S}_{5}$ & 2.665 & $\mathrm{~T}_{5}$ & 2.316 & 0.0320 \\
\hline $\mathrm{S}_{6}$ & 2.695 & $\mathrm{~T}_{6}$ & 2.335 & 0.0000 \\
\hline$S_{7}$ & 2.762 & $\mathrm{~T}_{7}$ & 2.628 & 0.0012 \\
\hline $\mathrm{S}_{8}$ & 2.937 & $\mathrm{~T}_{8}$ & 2.644 & 0.0000 \\
\hline$S_{9}$ & 2.965 & $\mathrm{~T}_{9}$ & 2.674 & 0.0029 \\
\hline$S_{10}$ & 3.146 & $\mathrm{~T}_{10}$ & 2.947 & 0.0165 \\
\hline
\end{tabular}

Table S4: Vertical excitation energies and oscillator strengths of PDI and PDI-Twisted geometries for four lowest singlet excited states at TDDFT/B3LYP/6-31G(d,p) level of theory.

\begin{tabular}{|c|c|c|c|c|}
\hline \multirow{2}{*}{ State } & \multicolumn{2}{|c|}{ Energy $(\mathbf{e V})$} & \multicolumn{2}{c|}{ Oscillator strength } \\
\cline { 2 - 5 } & PDI & PDI-Twisted & PDI & PDI-Twisted \\
\hline$S_{1}$ & 2.44 & 2.55 & 0.666 & 0.568 \\
\hline$S_{2}$ & 3.14 & 3.11 & 0.000 & 0.0017 \\
\hline$S_{3}$ & 3.15 & 3.14 & 0.000 & 0.0016 \\
\hline$S_{4}$ & 3.38 & 3.29 & 0.000 & 0.0016 \\
\hline
\end{tabular}

Table S5. Spin-orbit coupling values between $S_{1}$ and low-lying triplet states at PDI minimum and PDITwisted geometries evaluated at TDDFT/B3LYP/6-31G(d,p) level of theory.

\begin{tabular}{|c|c|c|}
\hline \multirow{2}{*}{ States } & \multicolumn{2}{|c|}{ SOC $\left(\mathbf{c m}^{-1}\right)$} \\
\cline { 2 - 3 } & PDI & PDI-Twisted \\
\hline$S_{1} / T_{2}$ & 0.007 & 0.526 \\
\hline$S_{1} / T_{3}$ & 0.007 & 2.419 \\
\hline$S_{1} / T_{4}$ & 3.07 & 0.182 \\
\hline
\end{tabular}


Table S6: A comparative account of populating the triplet excited state in PDIs by covalent methods.

\begin{tabular}{|c|c|c|c|c|c|}
\hline & Contributed by & Journal and Year & Molecule & $\Phi_{T}$ & $\overline{\tau_{\text {ISC }}}$ \\
\hline 1 & Castellano and coworkers & $\begin{array}{l}\text { J.Phys.Chem.A. 2009,113, } \\
\text { 5763-5768 }\end{array}$ & PDI-Pt-PDI & na & $\begin{array}{l}4.1-4.26 \\
\text { ps }\end{array}$ \\
\hline 2 & Wang, Fu and coworkers & $\begin{array}{l}\text { J. Phys. Chem. Lett. 2010, 1, 2499- } \\
2502\end{array}$ & DiPBI-1 & 0.94 & $610 \mathrm{ps}$ \\
\hline 3 & $\begin{array}{l}\text { Scholes, Seferos and } \\
\text { coworkers }\end{array}$ & $\begin{array}{l}\text { J.Phys.Chem. C 2014,118, } \\
\text { 9996-10004 }\end{array}$ & $\mathrm{S}_{3}$-PDI & na & $0.88 \mathrm{ps}$ \\
\hline 4 & $\begin{array}{l}\text { Steffen, Wurthner and } \\
\text { coworkers }\end{array}$ & $\begin{array}{c}\text { Angew.Chem.Int.Ed, 2015,54, } \\
\text { 1570-1573 }\end{array}$ & $\begin{array}{l}{[\mathrm{Ru}(\mathrm{bpy}) 2(\mathrm{ab}-} \\
\mathrm{PBI})][\mathrm{PF} 6] 2\end{array}$ & na & na \\
\hline 5 & Hariharan and coworkers & $\begin{array}{c}\text { J.Phys.Chem. C 2016,120, 8443- } \\
8450\end{array}$ & PDI-Br ${ }_{4}$ & 0.18 & na \\
\hline 6 & $\mathrm{Wu}, \mathrm{Fu}$ and coworkers & Chem. Eur. J, 2016,22,4717-4722 & MeS-PDI & 0.86 & $91 \pm 8$ ps \\
\hline 7 & Hariharan and coworkers & Current work & OBPDI & 0.97 & $14.20 \mathrm{ps}$ \\
\hline
\end{tabular}




\section{FIGURES}

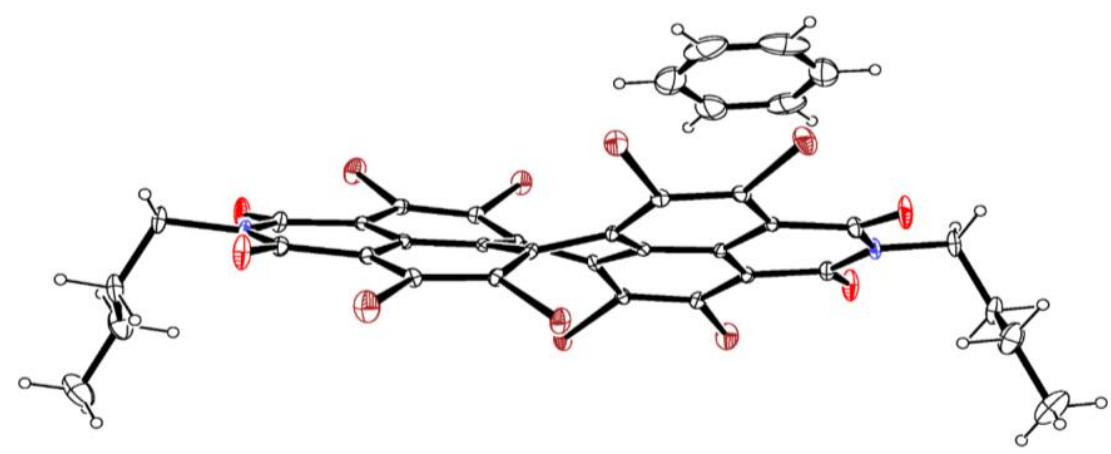

Figure S1: ORTEP image OBPDI crystallized from benzene.

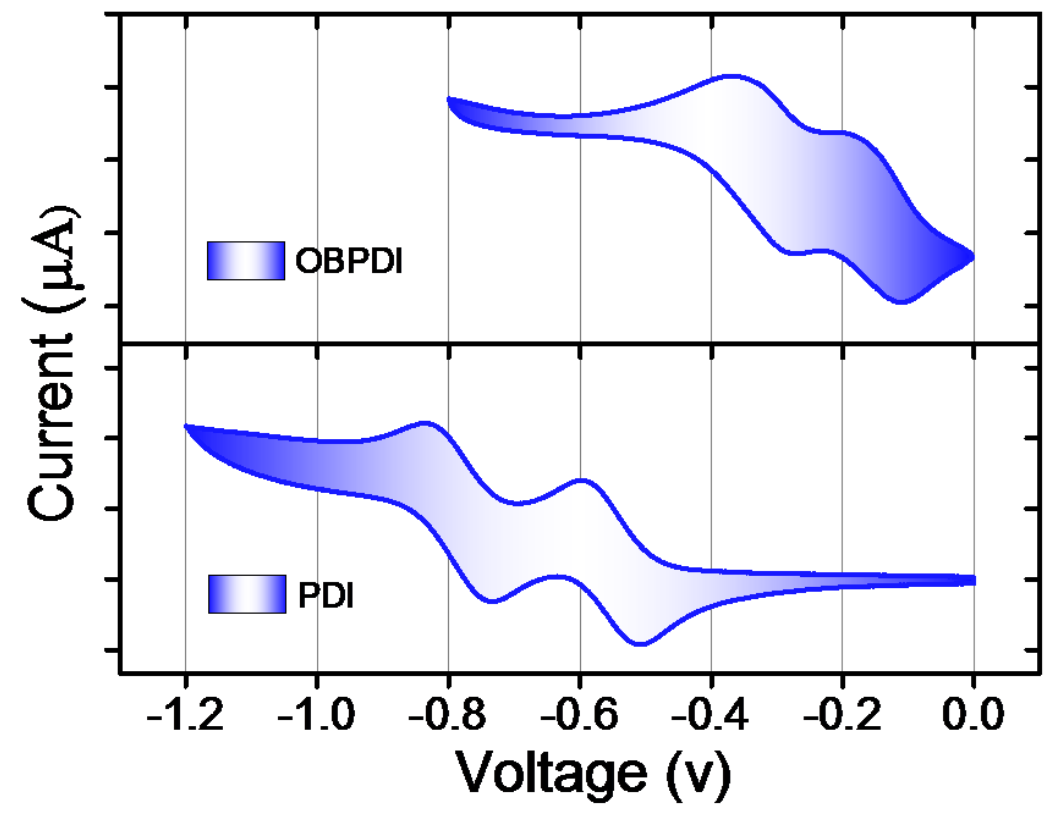

Figure S2: Cyclic Voltammogram of OBPDI and PDI. 


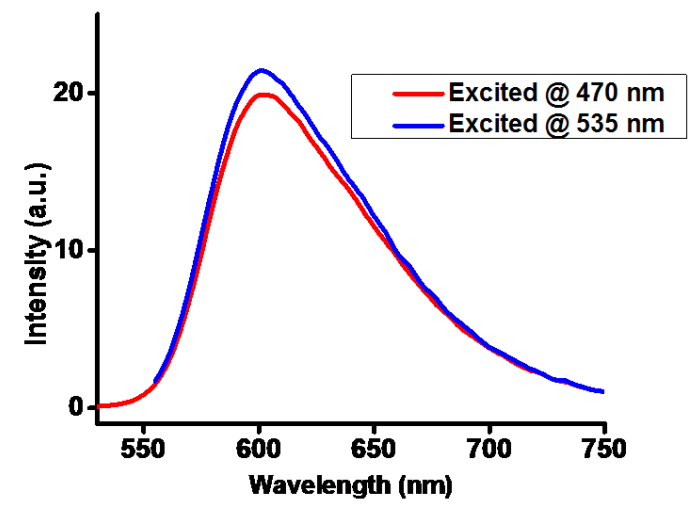

Figure S3: Fluorescence emission spectra of OBPDI.

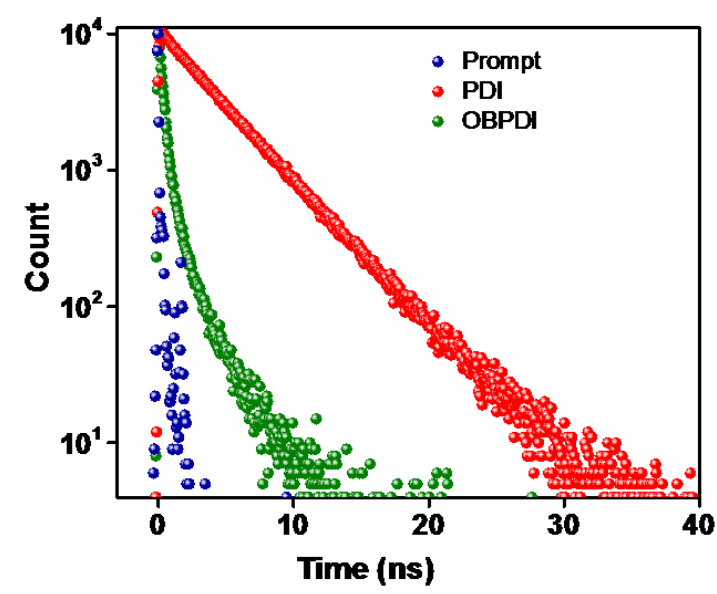

Figure S4: Fluorescence decay profiles of OBPDI and PDI.

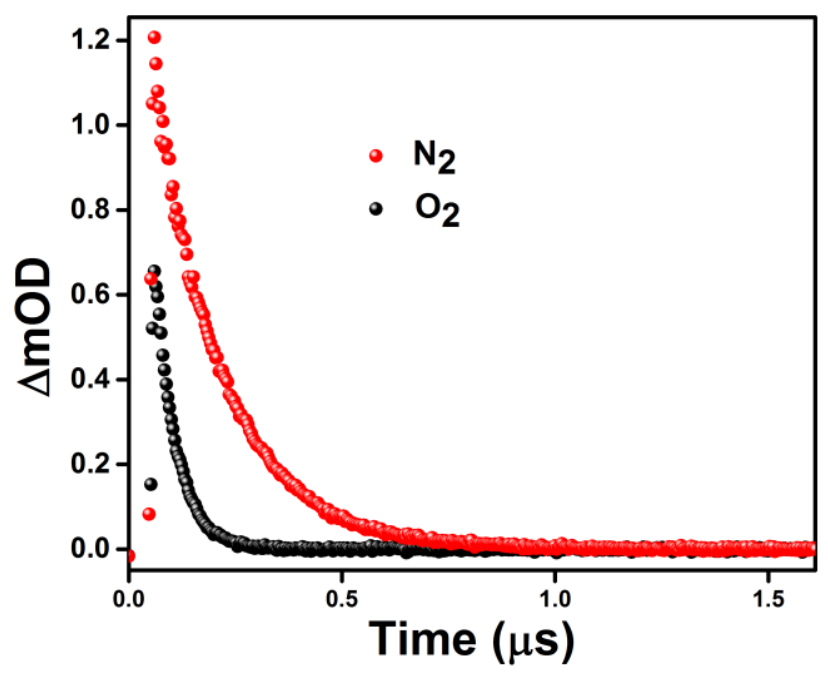

Figure S5: The decay traces at the triplet maxima under $\mathrm{N}_{2}$ and $\mathrm{O}_{2}$ purged conditions in OBPDI. 


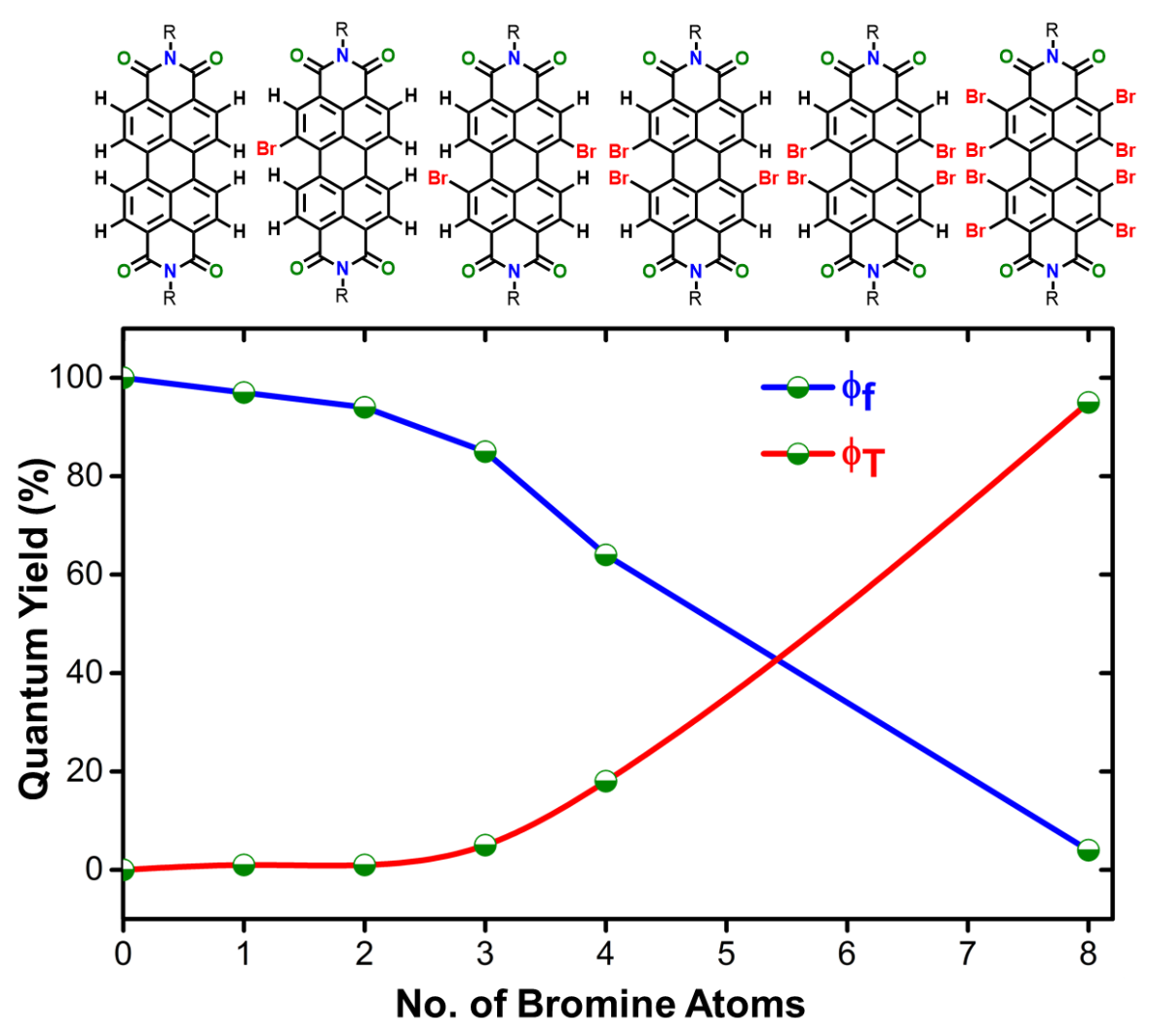

Figure S6: Graphical representation of trend in the fluorescence $\left(\Phi_{1}\right)$ and triplet $\left(\Phi_{1}\right)$ quantum yields with increase in the number of bromine atoms (from $\mathrm{Br}_{0}$-PDI ( 0 ; $\mathrm{x}$-axis), $\mathrm{Br}_{1}$-PDI ( 1 ; $\mathrm{x}$-axis), $\mathrm{Br}_{2}$-PDI (2; $\mathrm{x}$-axis), $\mathrm{Br}_{3}$-PDI (3; $\mathrm{x}$-axis), $\mathrm{Br}_{4}$-PDI (4; $\mathrm{x}$-axis) and OBPDI/Br 8 -PDI (8; $\mathrm{x}$-axis).

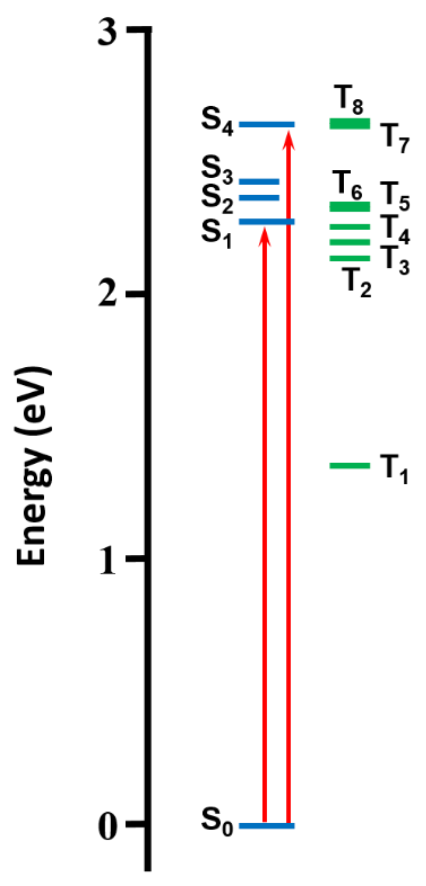


Figure S7: Graphical representation for vertical energies of lowest singlet and triplet excited states of OBPDI (computed at TDDFT/B3LYP/6-31G (d,p) level of theory).

\section{Cartesian Coordinates for Optimized Minima}

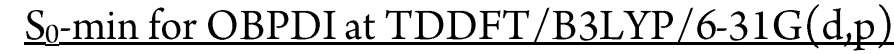

\begin{tabular}{|c|c|c|c|}
\hline $\mathrm{C}$ & -0.025825 & 0.357013 & -0.001925 \\
\hline $\mathrm{C}$ & -0.029449 & 0.096425 & 1.468608 \\
\hline $\mathrm{C}$ & 1.219932 & 0.004602 & 2.152578 \\
\hline $\mathrm{C}$ & 2.464240 & 0.014822 & 1.453688 \\
\hline $\mathrm{C}$ & 2.442227 & -0.017298 & -0.037517 \\
\hline $\mathrm{N}$ & 1.206611 & 0.207886 & -0.648722 \\
\hline $\mathrm{C}$ & 3.654467 & 0.038342 & 2.186985 \\
\hline $\mathrm{C}$ & 3.635510 & -0.162759 & 3.594039 \\
\hline $\mathrm{C}$ & 2.433142 & -0.399062 & 4.262482 \\
\hline $\mathrm{C}$ & 1.225687 & -0.101006 & 3.566967 \\
\hline $\mathrm{C}$ & 0.023931 & 0.089528 & 4.308210 \\
\hline $\mathrm{C}$ & 0.176831 & 0.367195 & 5.746678 \\
\hline $\mathrm{C}$ & 1.237092 & -0.311098 & 6.414730 \\
\hline $\mathrm{C}$ & 2.290615 & -0.884546 & 5.645831 \\
\hline $\mathrm{C}$ & 1.243672 & -0.414588 & 7.829273 \\
\hline $\mathrm{C}$ & 2.197306 & -1.270391 & 8.458068 \\
\hline $\mathrm{C}$ & 3.016766 & -2.073803 & 7.658351 \\
\hline C & 3.057278 & -1.882153 & 6.250482 \\
\hline C & -0.587516 & 1.262388 & 6.496844 \\
\hline $\mathrm{C}$ & -0.534869 & 1.246232 & 7.917259 \\
\hline C & 0.295246 & 0.339526 & 8.583363 \\
\hline C & 0.217812 & 0.114799 & 10.056014 \\
\hline $\mathrm{N}$ & 1.254165 & -0.625123 & 10.630049 \\
\hline $\mathrm{C}$ & 2.298094 & -1.261220 & 9.948087 \\
\hline C & -1.213505 & -0.042010 & 2.200293 \\
\hline C & -1.183209 & -0.050718 & 3.621377 \\
\hline $\mathrm{Br}$ & 4.068857 & -3.064017 & 5.168349 \\
\hline $\mathrm{Br}$ & 4.099835 & -3.442400 & 8.390764 \\
\hline $\mathrm{O}$ & -0.676518 & 0.535517 & 10.770904 \\
\hline C & 1.223043 & -0.723961 & 12.096991 \\
\hline $\mathrm{O}$ & 3.218418 & -1.767510 & 10.567161 \\
\hline $\mathrm{Br}$ & -1.612812 & 2.585507 & 5.608735 \\
\hline $\mathrm{Br}$ & -1.621245 & 2.481394 & 8.852938 \\
\hline $\mathrm{Br}$ & 5.332182 & 0.330730 & 1.361690 \\
\hline $\mathrm{Br}$ & 5.238648 & 0.006755 & 4.589941 \\
\hline $\mathrm{Br}$ & -2.897414 & -0.225496 & 1.355717 \\
\hline
\end{tabular}




\begin{tabular}{|c|c|c|c|}
\hline $\mathrm{Br}$ & -2.776870 & -0.372538 & 4.594925 \\
\hline $\mathrm{O}$ & 3.421081 & -0.219672 & -0.736649 \\
\hline $\mathrm{C}$ & 1.234410 & 0.298121 & -2.116285 \\
\hline $\mathrm{O}$ & -1.016774 & 0.680849 & -0.634167 \\
\hline $\mathrm{H}$ & 1.909327 & 1.099338 & -2.423126 \\
\hline $\mathrm{H}$ & 0.222944 & 0.500556 & -2.455934 \\
\hline $\mathrm{H}$ & 2.091294 & -1.296340 & 12.409922 \\
\hline $\mathrm{H}$ & 0.299278 & -1.213553 & 12.410026 \\
\hline $\mathrm{H}$ & 1.605436 & -0.640075 & -2.532413 \\
\hline $\mathrm{H}$ & 1.245477 & 0.276149 & 12.533902 \\
\hline
\end{tabular}

$\underline{S}_{0}-$ min for PDI at TDDFT/B3LYP/6-31G $(d, p)$

\begin{tabular}{|c|c|c|c|}
\hline $\mathrm{C}$ & -1.210696 & 0.539200 & 3.531088 \\
\hline $\mathrm{C}$ & 0.095130 & 0.435950 & 4.228210 \\
\hline $\mathrm{C}$ & 1.232552 & -0.101156 & 3.577775 \\
\hline $\mathrm{C}$ & 1.125126 & -0.547989 & 2.238674 \\
\hline $\mathrm{C}$ & -0.162600 & -0.460018 & 1.510026 \\
\hline $\mathrm{N}$ & -1.251553 & 0.079584 & 2.205702 \\
\hline $\mathrm{C}$ & 2.233665 & -1.073686 & 1.598743 \\
\hline $\mathrm{C}$ & 3.459258 & -1.166048 & 2.266704 \\
\hline C & 3.614786 & -0.738585 & 3.586856 \\
\hline $\mathrm{C}$ & 2.480835 & -0.191823 & 4.267800 \\
\hline $\mathrm{C}$ & 2.563494 & 0.266511 & 5.621009 \\
\hline $\mathrm{C}$ & 3.847701 & 0.172863 & 6.331247 \\
\hline $\mathrm{C}$ & 4.981765 & -0.373484 & 5.650156 \\
\hline $\mathrm{C}$ & 4.899223 & -0.831382 & 4.296791 \\
\hline $\mathrm{C}$ & 6.230050 & -0.464123 & 6.340175 \\
\hline $\mathrm{C}$ & 7.367697 & -1.000412 & 5.689457 \\
\hline $\mathrm{C}$ & 7.265439 & -1.436960 & 4.380049 \\
\hline $\mathrm{C}$ & 6.047653 & -1.352101 & 3.697178 \\
\hline $\mathrm{C}$ & 4.002899 & 0.599131 & 7.651822 \\
\hline $\mathrm{C}$ & 5.228482 & 0.506738 & 8.319799 \\
\hline $\mathrm{C}$ & 6.337280 & -0.018003 & 7.679530 \\
\hline $\mathrm{C}$ & 7.625190 & -0.105270 & 8.407935 \\
\hline $\mathrm{N}$ & 8.714135 & -0.644924 & 7.712282 \\
\hline $\mathrm{C}$ & 8.673367 & -1.104234 & 6.386788 \\
\hline $\mathrm{C}$ & 0.197714 & 0.873687 & 5.537198 \\
\hline $\mathrm{C}$ & 1.415437 & 0.788605 & 6.220145 \\
\hline $\mathrm{O}$ & 7.757473 & 0.270742 & 9.565221 \\
\hline $\mathrm{C}$ & 9.981924 & -0.724703 & 8.446521 \\
\hline $\mathrm{O}$ & 9.667580 & -1.564744 & 5.841407 \\
\hline $\mathrm{O}$ & -0.294526 & -0.834691 & 0.352265 \\
\hline $\mathrm{C}$ & -2.519162 & 0.160050 & 1.471230 \\
\hline $\mathrm{O}$ & -2.204771 & 1.000227 & 4.076284 \\
\hline $\mathrm{H}$ & 5.331705 & 0.842693 & 9.345737 \\
\hline $\mathrm{H}$ & 3.162179 & 1.016307 & 8.192158 \\
\hline
\end{tabular}




\begin{tabular}{|c|c|c|c|}
\hline $\mathrm{H}$ & 1.447725 & 1.143521 & 7.242783 \\
\hline $\mathrm{H}$ & 4.299699 & -1.584228 & 1.726722 \\
\hline $\mathrm{H}$ & 2.130249 & -1.410354 & 0.573058 \\
\hline $\mathrm{H}$ & 8.147335 & -1.844473 & 3.898368 \\
\hline $\mathrm{H}$ & 6.015665 & -1.705962 & 2.674172 \\
\hline $\mathrm{H}$ & 10.289374 & 0.274251 & 8.762372 \\
\hline $\mathrm{H}$ & 10.722043 & -1.161680 & 7.781369 \\
\hline $\mathrm{H}$ & -3.260150 & 0.593774 & 2.137543 \\
\hline $\mathrm{H}$ & -2.824891 & -0.838316 & 1.151960 \\
\hline $\mathrm{H}$ & 9.853678 & -1.340768 & 9.338796 \\
\hline $\mathrm{H}$ & -2.391631 & 0.779310 & 0.581034 \\
\hline $\mathrm{H}$ & -0.683963 & 1.282063 & 6.018568 \\
\hline
\end{tabular}

\section{REFERENCES}

(1) Philip, A. M.; Gudem, M.; Sebastian, E.; Hariharan, M. Decoding the Curious Tale of Atypical Intersystem Crossing Dynamics in Regioisomeric Acetylanthracenes. J. Phys. Chem. A 2019, 123, $6105-6112$.

(2) Rajagopal, S. K.; Philip, A. M.; Nagarajan, K.; Hariharan, M. Progressive Acylation of Pyrene Engineers Solid State Packing and Colour via C-H...H-C, C-H...O and $\pi-\pi$ Interactions. Chem. Commun. (Camb). 2014, 50, 8644-8647.

(3) Wu, Y.; Zhen, Y.; Ma, Y.; Zheng, R.; Wang, Z.; Fu, H. Exceptional Intersystem Crossing in Di(Perylene Bisimide)s: A Structural Platform toward Photosensitizers for Singlet Oxygen Generation. J. Phys. Chem. Lett. 2010, 1, 2499-2502.

(4) Kumar, Y.; Kumar, S.; Kumar Keshri, S.; Shukla, J.; Singh, S. S.; Thakur, T. S.; Denti, M.; Facchetti, A.; Mukhopadhyay, P. Synthesis of Octabromoperylene Dianhydride and Diimides: Evidence of Halogen Bonding and Semiconducting Properties. Org. Lett. 2016, 18, 472-475. 